J. Lake Sci. (湖泊科学), $2007,19(1): 58-62$

http:// www. jlakes. org. E-mail: jlakes@ niglas. ac.cn

(c) 2007 by Journal of Lake Sciences

\title{
浙江东钱湖底泥污染物分布特征与评价
}

\author{
程南宁, 李 巍, 再光兴, 杜心慧, 曹 卉 \\ (上海勘测设计研究院,上海 200434)
}

\begin{abstract}
摘 要: 对东钱湖底泥做了全面调查,测定了底泥粒度组成、有机质、TN、TP 及重金属含量, 分析了底泥沉积特性及污染 物分布特征,分别采用潜在生态危害指数 $(R I)$ 法和相对背景值法对重金属污染风险和有机污染及营养盐污染进行了评 价. 研究结果表明: 东钱湖底泥组成以细颗粒为主, 重金属含量除镉污染达到中等生态危害程度外,其余均处于较低水 平, 湖区底泥中有机污染和氮污染问题非常突出. 为有效治理东钱湖内源污染, 有必要开展包括生态疏浚在内的内源污 染治理措施. 研究为进一步开展东钱湖水环境治理提供了理论依据.
\end{abstract}

关键词: 东钱湖; 底泥; 分布特征; 潜在生态危害指数 $(R I)$; 相对背景值; 评价

\section{Characteristics and risk evaluation of pollutants in sediments of Lake Dongqian, Zhejiang Province}

CHENG Nanning, LI Wei, RAN Guangxing, DU Xinghui \& CAO Hui (Shanghai Investigation, Design \& Research Institute, Shanghai 200434,P. R. China)

\begin{abstract}
Investigations were carried out in order to obtain sizes grades and the content of organic, total nitrogen, total phosphorus and heavy metals in sediments of Lake Dongqian (a lake in Ningbo city). Sedimentation character and pollutant distribution characteristics were analyzed in the article. The results indicated that the chief component of sediments was fine particle. The concentrations of most heavy metals were low except for $\mathrm{Cd}$, which showed medium ecological risk. Heavy metals pollution level was slight as evaluated by Hakanson potential ecological risk index method, whereas organic and nitrogen pollution was severe compared with relative environment baseline such as in bottom layer sedimentary and Taihu Lake sediment. Heavy pollution sediments in upper layer were also noted. In order to efficiently control the internal source pollution, it is necessary to employ internal source pollution control measures such as ecological dredging. The research provides theoretical support for Lake Dongqian water environment treatment.
\end{abstract}

Keywords: Lake Dongqian; sediment; pollution characteristics; potential ecological risk index; relative baseline; evaluation

东钱湖位于宁波市东南郊,是浙江省第一大天然淡水湖. 全湖由谷子湖、北湖和南湖组成,东西宽约 $6.5 \mathrm{~km}$, 南北长约 $8.5 \mathrm{~km}$, 湖面面积 $19.9 \mathrm{~km}^{2}$, 平均水深 $2.0 \mathrm{~m}$, 换水周期约为 $280 \mathrm{~d}$, 是典型的盆状浅水 湖泊.

随着人口数量的增加、工农业的发展以及城市化进程的加剧,东钱湖沿湖地区人湖污染负荷逐年增加， 导致湖区水体和底泥中的污染物不断累积, 湖泊富营养化程度日趋严重,生物种群单一化趋势明显, 生态系 统正日益退化. 作为宁波市规划中的后备水源地和国家级生态型旅游度假区,东钱湖水环境治理问题十分 迫切.

底泥作为湖泊中污染物的蓄积库和内污染源早已为人们所认识 ${ }^{[1-4]}$. 即使在外源污染得到控制的条 件下,底泥中蓄积的污染物质也会向上覆水中产生释放,影响湖水水质. 东钱湖目前正在采取多种措施集

* 建设部科技攻关项目 $(03-2-069)$ 资助. 2006-01-20 收稿;2006-04-29 收修改稿. 程南宁, 男, 1980 年生, 硕 士;E-mail:chnanning@163.com. 
中治理人湖外源污染, 内源治理也正在进行中. 鉴于东钱湖历史上尚未对底泥开展过研究调查, 因此在外 源治理的同时非常有必要对湖泊的内源污染进行调查和研究.

本文通过对东钱湖底泥现状开展调查,分析了东钱湖底泥中的粒度组成、有机质、TN、TP 以及重金属含 量的分布及相关特征;采用潜在生态危害指数 $(R I)$ 法评价了底泥中重金属的潜在危害性. 采用相对背景值 法评价了底泥中有机质及营养盐的污染程度. 研究为东钱湖进一步开展内源污染治理工作提供了理论和 实验依据.

\section{1 材料与方法}

\section{1 采样点布设}

监测样点采用 GPS 系统精确定位. 底泥监测点基本按每 $1 \mathrm{~km} \times 1 \mathrm{~km}$ 网格范围 1 个点的密度布设,考虑 河流出、人湖口、取水口、航道线及污染较严重区域局部加密布设. 整个湖区共布设粒度分析采样点 10 个, 柱状分层采样点 21 个; 在人湖河口及沿岸带布设表层采样点 12 个. 采样点具体位置见图 1.

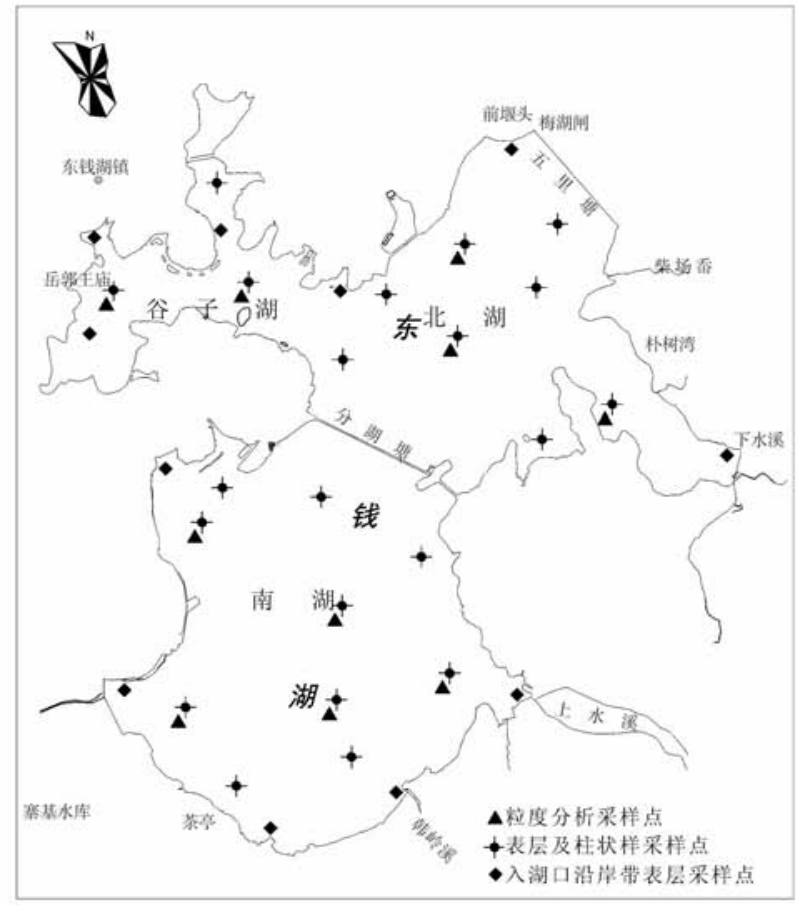

图 1 东钱湖采样点分布

Fig. 1 Distribution of sampling sites in Lake Dongqian

\section{2 采样方法}

采用彼得森采样器采集底泥样品. 粒度分析样分 $0-20 \mathrm{~cm} 、 20-100 \mathrm{~cm} 、 100 \mathrm{~cm}$ 以下三层留取样品. 柱 状样根据不同采样点底泥淤积厚度情况, 分别留取 0-10 cm、10-20 cm、20-40 cm、40-60 cm、60-100 $\mathrm{cm} 、 100 \mathrm{~cm}$ 以下层泥样; 局部点位自然分层明显, 则结合自然分层进行留样. 表层样留取 $0-20 \mathrm{~cm}$ 层泥样. 样品经现场处理后带回实验室分析.

\section{3 分析项目及测定方法}

底泥柱状样及表层底泥样风干后, 分别测定样品中粒度组成、营养物质含量和重金属含量. 参照《湖泊 富营养化调查规范 $\rangle^{[5]}$, 底泥粒度组成采用笁分法与消光法分析; 有机质、TN、TP 含量分别采用重铬酸钾容 量法、高氯酸 - 硫酸消化法、高氯酸 - 硫酸酸溶 - 钼锑抗比色法分析 $; \mathrm{Hg}$ 含量采用冷原子光度法分析, $\mathrm{Cd}$ 
和 $\mathrm{Pb}$ 含量采用石墨炉原子吸收光度法分析, $\mathrm{Cr}$ 和 $\mathrm{Cu}$ 含量采用火焰原子吸收光度法分析, $\mathrm{As}$ 含量采用二乙 基二硫代氨基甲酸银光度法测定.

\section{2 结果与讨论}

\section{1 基本物理性状特征}

粒度分析结果表明:东钱湖底泥粒径组成以粉粒、粘粒等粒径较小的细颗粒为主. 不同泥层的底泥中 5 $-50 \mu \mathrm{m}$ 的粉粒平均含量为 $68.50 \%$; 其次为 $1-5 \mu \mathrm{m}$ 的粗粘粒, 平均含量为 $24.46 \% ; 1 \mu \mathrm{m}$ 以下、50 -1000 $\mu \mathrm{m} 、 1 \mathrm{~mm}$ 以上粘粒平均含量分别为 $6.20 \% 、 1.04 \% 、 0 \%$ (表 1). 底泥中颗粒物中值粒径 $d$ 为 $9.13 \mu \mathrm{m}$. 各 湖区中下层的中值粒径差异不大,但从表层 $(0-20 \mathrm{~cm})$ 来看则有显著不同. 谷子湖和北湖中值粒径较南湖 细, 反映两湖区表层沉积物粘土质底泥含量较高. 由于表层 $0-20 \mathrm{~cm}$ 底泥主要是近代沉积物,因此可初步 判断北部湖区受人类活动影响较南部湖区大.

表 1 东钱湖底泥中重金属含量与标准值对照表 $(\mathrm{mg} / \mathrm{kg})$

Tab. 1 Comparison of heavy metal concentrations of sediments in Lake Dongqian with different standards $(\mathrm{mg} / \mathrm{kg})$

\begin{tabular}{ccccccc}
\hline & $\mathrm{Hg}$ & $\mathrm{As}$ & $\mathrm{Cu}$ & $\mathrm{Cr}$ & $\mathrm{Pb}$ & $\mathrm{Cd}$ \\
\hline 最大值 & 0.089 & 17.65 & 99.6 & 84.3 & 78.1 & 1.46 \\
$0-10 \mathrm{~cm}$ 层 & 0.036 & 6.19 & 29.3 & 50.2 & 54.6 & 1.04 \\
$10-20 \mathrm{~cm}$ 层 & 0.034 & 6.68 & 27.8 & 45.2 & 45.8 & 0.93 \\
$20-40 \mathrm{~cm}$ 层 & 0.028 & 7.83 & 25.9 & 45.6 & 39.6 & 0.99 \\
$40-60 \mathrm{~cm}$ 层 & 0.025 & 8.05 & 27.7 & 53.7 & 39.8 & 1.00 \\
$60-100 \mathrm{~cm}$ 层 & 0.026 & 10.72 & 29.9 & 58.3 & 37.4 & 0.98 \\
$100 \mathrm{~cm}$ 以下 & 0.025 & 11.70 & 31.5 & 61.9 & 40.0 & 0.98 \\
全湖平均值 & 0.030 & 8.14 & 28.4 & 51.2 & 43.1 & 0.99 \\
土壤背景值 & 0.048 & 7.4 & 23.0 & 70.1 & & \\
\hline
\end{tabular}

调查共采集底泥垂直分层样 112 个,底泥表层样 12 个. 从底泥垂直分层特征来看,东钱湖底泥沉积自 上而下基本呈现流泥 - 淤泥质粘土 - 淤泥 - 淤泥质粘土 - 粘土的较为显著的分层结构. 表层 $(0-20 \mathrm{~cm})$ 为灰色、流塑态、含有大量有机质、密度很小的流泥层, 在风浪等外力扰动条件下极易发生流动和再悬浮. 中间 $(20-60 \mathrm{~cm})$ 为灰色、流塑态、含少量腐殖物的淤泥和淤泥质粘土层. 从含水量、空隙比、底泥密度比 较, 表层以下的第二层和第四层淤泥质粘土层间夹有一层相对松软的淤泥层. 底层 (60 cm 以下) 为呈黄褐 等色、可塑态、高韧性、土层密实的粘土层. 底泥垂直剖面基本符合沉积学标准垂直剖面.

\section{2 重金属含量分布特征及评价}

从总体分布特征来看,谷子湖底泥中重金属的含量普遍略高于北湖、南湖. 各湖区重金属含量垂向无 明显的分布规律. 全湖录、铜、砷含量的最大值均出现在谷子湖的同一监测点,说明谷子湖底泥中的重金属 来源具有一定的同源性,可能与湖边工厂企业的生产废水排放有关.

相对于宁波地区土壤背景值 ${ }^{[6]}$ 而言, 全湖底泥中除镉含量较高外, 其余重金属含量均处于较低水平 （表 1 ）; 重金属含量最大值点均分布在表层 $0-40 \mathrm{~cm}$ 范围内.

表 2 东钱湖底泥中重金属的潜在生态危害指数 (Eir) 和危害指数 $(R I)$

Tab. 2 Potential ecological risk factors ( Eir) and risk indices $(R I)$ of heavy metals in sediments of Lake Dongqian

\begin{tabular}{cccccccc}
\hline 湖区 & $\mathrm{Hg}$ & $\mathrm{As}$ & $\mathrm{Cu}$ & $\mathrm{Cr}$ & $\mathrm{Pb}$ & $\mathrm{Cd}$ & $R I$ \\
\hline 谷子湖 & 6.88 & 6.41 & 6.23 & 1.78 & 9.22 & 60.60 & 91.13 \\
北湖 & 4.80 & 4.97 & 4.48 & 1.76 & 8.66 & 58.80 & 83.47 \\
南湖 & 4.32 & 5.59 & 4.63 & 1.65 & 8.48 & 59.40 & 84.08 \\
全湖 & 4.80 & 5.43 & 4.73 & 1.71 & 8.62 & 59.40 & 84.69 \\
\hline
\end{tabular}


重金属潜在生态危害采用瑞典科学家 Hakanson 提出的潜在生态危害指数 $(R I)$ 法 $^{[7]}$ 进行评价. 评价结 果 (表 2) 显示东钱湖沉积底泥中重金属生态危害程度都较小,各湖区差异性不大. 从单项污染指数来看, 镉的生态危害程度远高于其它元素,达到中等生态危害的污染程度.

\section{3 有机质及营养盐含量分布特征及评价}

全湖底泥有机质含量在 $0.94 \%-14.41 \%$ 之间, 平均含量为 $4.24 \%$; 各湖区中含量最高的是南湖, 其次为 北湖和谷子湖. TP 含量在 $0.014 \%-0.086 \%$ 之间,平均为 $0.035 \%$; 各湖区中含量最高的是谷子湖,其次为南 湖和北湖. TN 含量在 $0.04 \%-0.76 \%$ 之间, 平均含量为 $0.19 \%$, 各湖区含量差别不大. 就三个湖区中表层底 泥中的物质含量来看,南湖有机质平均含量较高, 谷子湖 TP 平均含量较高, TN 平均含量各湖区基本接近.

各湖区的污染物含量垂直分布特征基本一致(图 2). 有机质和 TN 含量垂向均呈现随深度增加而减小 的趋势,TP 含量垂直分布自表层至下呈现下降趋势, 在 $40-60 \mathrm{~cm}$ 处存在极小值拐点,拐点以下有逐渐上 升的趋势. 各湖区的有机物、TN、TP 污染严重的层位主要集中在表层. 由于缺乏明确的沉积物质量标准, 本文利用相对背景值的比较方法, 采用湖泊下层沉积以及相近湖泊底泥中污染物含量做评价的参比值, 对 东钱湖表层底泥中有机污染及营养物质含量进行评价.


图 2 东钱湖底泥中有机质、TN、TP 含量垂直分布

Fig. 2 The distribution of organic, TN and TP Content in the sediments of Lake Dongqian

根据湖泊沉积特性和湖区地质勘察资料分 析,湖区受人类活动影响严重的近代沉积主要 集中在表层 $30 \mathrm{~cm}$ 以内, 考虑风浪扰动以及污 染物迁移, 可认为 $50 \mathrm{~cm}$ 以下的沉积是受人类 活动影响较小的古代沉积物, 其物质含量可以 作为湖泊沉积物的相对背景值. 本文根据底泥 柱状样分层留样特征, 采用全湖 $60 \mathrm{~cm}$ 以下底 泥样品平均值作为参比值, 同时对比太湖污染 严重的五里湖、梅梁湖北部湖区、竺山湖以及东 太湖四个湖区表层底泥中污染物含量 (1), 对东 钱湖表层底泥的污染程度进行评价.

评价结果 (表 3 ) 显示东钱湖底泥中 TP 含 量总体处于较低水平, 但有机污染和氮污染问 题非常突出. 相对于湖区 $60 \mathrm{~cm}$ 以下沉积层的 参比值,东钱湖表层底泥中全湖有机质和 TN
表 3 东钱湖表层底泥有机质、TN、TP 含量与参照值的对照 Tab. 3 Comparison of organic, TN and TP concentrations in sediments of Lake Dongqian with different standards

\begin{tabular}{cccc}
\hline 湖区 & 有机质 $(\%)$ & $\mathrm{TP}(\%)$ & $\mathrm{TN}(\%)$ \\
\hline 谷子湖 & 4.94 & 0.060 & 0.27 \\
北湖 & 5.15 & 0.034 & 0.27 \\
南湖 & 6.58 & 0.035 & 0.28 \\
东钱湖平均 & 5.86 & 0.037 & 0.28 \\
东钱湖底层参比值 & 2.19 & 0.045 & 0.094 \\
五里湖 & 4.39 & 0.184 & 0.186 \\
梅梁湖北部 & 2.23 & 0.088 & 0.147 \\
竺山湖 & 2.07 & 0.115 & 0.126 \\
东太湖 & 4.44 & 0.049 & 0.258 \\
全太湖平均 & 1.56 & 0.051 & 0.098 \\
\hline
\end{tabular}


平均值分别超过参比值 2.68 和 2.98 倍; 相对太湖表层底泥监测结果, 东钱湖表层底泥有机质和 TN 含量分 别是全太湖平均值的 3.76 和 2.86 倍, 比太湖重污染的五里湖和养殖负荷极高的东太湖还要高, 可能与湖 周农业过度使用氮肥以及过去的围网养殖等有关.

\section{3 小结}

东钱湖底泥的调查分析结果表明: 湖区底泥组成以细颗粒为主;底泥沉积自上而下呈现流泥 - 淤泥质 粘土 - 淤泥 - 淤泥质粘土 - 粘土的较为显著的分层结构, 表层以下的第二层和第四层淤泥质粘土层之间夹 有相对松软的淤泥层,这种特殊的夹层结构对生态疏浚时疏浚深度的确定有参考意义; 重金属含量除镉污 染达到中等生态危害程度外, 其余均处于较低水平; 湖区底泥中有机污染和氮污染问题非常突出, 污染严重 的层位主要集中在表层沉积中.

因此,为有效治理东钱湖内源污染,有必要开展包括生态疏浚在内的内源污染治理措施,并进一步开展 底泥释放试验研究. 在进行生态疏浚研究时应根据东钱湖底泥的颗粒组成、沉积特性及污染物分布特征确 定疏浚范围、疏浚深度、疏浚过程中的余水处理方案以及疏浚后的底泥处置利用方式.

致谢: 本文的内容参考了《东钱湖综合整治可行性研究报告》的有关成果,在此谨向该报告的有关作者表示 感谢!

\section{4 参考文献}

[1] 金相灼. 湖泊富营养化控制和管理技术. 北京: 化学工业出版社,2001.

[2] Welch E B, Cooke G D. Effectiveness and longevity of phosphorus inactivation with alum. Lake and Reservoir Management, 1999, 15: 5-27.

[3] Søndergaard M, Jensen J P, Jeppesen E. Internal phosphorus loading in shallow Danish lakes. Hydrobiologia, 1999, 408/409: $145-152$.

[4] Sondergaard M, Jensen J P, Jeppesen E. Retention and internal loading of phosphorus in shallow, eutrophic lakes. The Scientific World, 2001, 1: $427-442$.

[5] 金相灿, 屠清瑛. 湖泊富营养化调查规范 (第二版). 北京: 中国环境科学出版社, 1990.

[6] 李 健, 郑春江. 环境背景值数据手册. 北京: 中国环境科学出版社, 1989.

[7] Hakanson L. An ecological risk index for aquatic pollution control of sediment. Water Res, 1980, 14(8): 975 - 1001. 